Received: 13 March 2019

Accepted: 7 June 2019

Published online: 03 July 2019
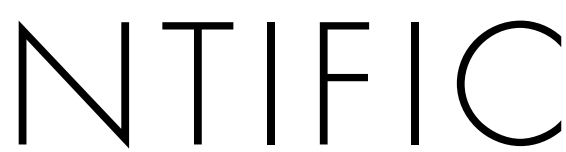

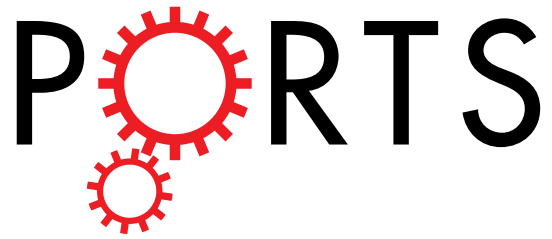

\title{
On the potential of in vitro organ-chip models to define temporal pharmacokinetic- pharmacodynamic relationships
}

Christopher W. McAleer ${ }^{1}$, Amy Pointon ${ }^{2}$, Christopher J. Long ${ }^{1}$, Rocky L. Brighton ${ }^{1}$, Benjamin D. Wilkin ${ }^{1}$, L. Richard Bridges ${ }^{1}$, Narasimham Narasimhan Sriram ${ }^{1}$, Kristin Fabre ${ }^{3}$, Robin McDougall ${ }^{3}$, Victorine P. Muse $\mathbb{D}^{3}{ }^{3}$, Jerome T. Mettetal ${ }^{3}$, Abhishek Srivastava ${ }^{2}$, Dominic Williams ${ }^{2}$, Mark T. Schnepper ${ }^{4}$, Jeff L. Roles ${ }^{1}$, Michael L. Shuler ${ }^{1}$, James J. Hickman ${ }^{1,4}$ \& Lorna Ewart ${ }^{2}$

Functional human-on-a-chip systems hold great promise to enable quantitative translation to in vivo outcomes. Here, we explored this concept using a pumpless heart only and heart:liver system to evaluate the temporal pharmacokinetic/pharmacodynamic (PKPD) relationship for terfenadine. There was a time dependent drug-induced increase in field potential duration in the cardiac compartment in response to terfenadine and that response was modulated using a metabolically competent liver module that converted terfenadine to fexofenadine. Using this data, a mathematical model was developed to predict the effect of terfenadine in preclinical species. Developing confidence that microphysiological models could have a transformative effect on drug discovery, we also tested a previously discovered proprietary AstraZeneca small molecule and correctly determined the cardiotoxic response to its metabolite in the heart:liver system. Overall our findings serve as a guiding principle to future investigations of temporal concentration response relationships in these innovative in vitro models, especially, if validated across multiple time frames, with additional pharmacological mechanisms and molecules representing a broad chemical diversity.

Integrative pharmacology is a discipline that builds an understanding of the inter-relationship between pharmacokinetics (PK), the drug's time course for absorption, distribution, metabolism and excretion and pharmacodynamics (PD), the biological effect of a drug. In drug discovery, this multi-variate approach guides medicinal chemists to modify structural properties of a drug molecule to improve its chance of becoming a medicine in a process known as "lead optimization". Pharmacological effect is driven by drug concentration however the seminal work of Segre ${ }^{1}$ and Sheiner et al. ${ }^{2}$, identified that the maximum drug effect is not always driven by the peak drug concentration but that in some cases time is a critical factor influencing drug effect, especially in in vivo studies. This is known as hysteresis and is driven by limited access to the site of drug action or slow receptor kinetics. In the main, current drug discovery programs are only able to truly investigate concentration-effect-time relationships during the advanced stages of the preclinical program. At this stage there are typically between 1 and 3 potential drug candidates progressed to animal studies to confirm efficacy and safety. It is therefore costly and time consuming to discover that such potential candidates may have poor therapeutic qualities preventing their onward progression, not to mention the significant limitations of extrapolating animal data to humans because drug behavior in animals can be dramatically different to that in humans.

Over the last two decades there has been an explosion in the development of microphysiological systems (MPS) or "body-on-a-chip" models ${ }^{3,4}$. These microengineered in vitro models aim to recreate the in vivo tissue

${ }^{1}$ Hesperos, Inc., 3259 Progress Dr., Room 158, Orlando, FL, 32826-3230, USA. 2Drug Safety and Metabolism, IMED Biotech Unit, AstraZeneca, Cambridge, UK. ${ }^{3}$ Drug Safety and Metabolism, IMED Biotech Unit, AstraZeneca, Waltham, USA. ${ }^{4}$ NanoScience Technology Center, 12424 Research Parkway, Suite 400, Orlando, FL, 32826, USA. Christopher W. McAleer and Amy Pointon contributed equally. Correspondence and requests for materials should be addressed to J.J.H. (email: jhickman@hesperosinc.com) or L.E. (email: lorna.ewart@astrazeneca.com) 
microenvironment enabling cells, typically human in origin, to maintain viability and function. The hype that these systems could recapitulate organ function ${ }^{5}$ is gradually being replaced by hope that such systems might enable more eloquent models for drug discovery reducing the reliance on animals which has ethical, monetary, time and translational advantages.

Using a 'heart-on-a-chip' that was fluidically connected to a 'liver-on-a-chip' we set out to discover whether it was possible to derive in vitro temporal PKPD relationships with the well characterized small molecule drug terfenadine. This multi-organ platform utilized a pumpless system and serum-free medium with interconnected compartments ${ }^{6,7}$. The absence of a pump and the accompanying tubing enabled a bubble free, low volume system providing the ability to detect metabolite formation that might otherwise be diluted in higher-volume systems ${ }^{8}$. The sinusoidal oscillatory rocking profile for driving fluid flow was designed via a transient flow model driven by gravitational acceleration to produce shear stresses on the cellular layers of no more than 0.05 dynes/ $\mathrm{cm}^{2}$ (within acceptable physiological ranges) ${ }^{8,9}$ throughout the rocking profile, as described previously ${ }^{6}$. This PK model additionally includes parameters for convective transport of compounds throughout the system (mixing) and compound-specific adsorption to the housing materials and has been used for both hydrophobic and hydrophilic compounds ${ }^{7}$. Currently, the adsorption is determined experimentally for each compound, though this framework is expected to be expanded to create a prediction of the adsorption based on the physical characteristics of the compounds. This PK model is used to inform our predictive PKPD model and the effect of metabolites on toxicity in multi-organ systems has also been explored at single timepoints in this model ${ }^{10,11}$. The functional cardiac system utilized patterned human cardiomyocytes that had previously been shown to allow non-invasive measurements of field potential duration (FPD), conduction velocity and beat rate ${ }^{12}$.

We reproduced the terfenadine-induced increase in QT-interval in the heart only model and demonstrated that in the presence of a metabolically competent liver compartment, this effect was diminished. We also discovered that this pharmacodynamic effect was driven by the intracellular drug concentration in cardiomyocytes, consistent with the intracellular binding site of terfenadine to the hERG channel ${ }^{13,14}$. Furthermore, we have been able to build a mathematical model that predicts drug response in this MPS model and additionally that these approaches can also be used to qualitatively predict the effect of terfenadine in preclinical species. Finally, we demonstrated the value of MPS models in drug discovery by testing an AstraZeneca proprietary small molecule which was identified as having a hERG liability, due to the formation of a hERG active metabolite, during the final stages of lead optimization. Taken together, these results provide the first description of integrative pharmacology in MPS models and builds confidence towards the transformative effect these models can have on drug discovery.

\section{Results}

Characteristics of heart:liver and heart only MPS models. Figure 1 shows the composition of the heart:liver and heart only models. Each model encompassed a microfluidic system (total volume $2 \mathrm{~mL}$ ), 2 media chambers and 2 (heart model) or 3 (heart:liver model) cellular chambers ( 1 liver and 2 cardiac). These cellular chambers contain electrodes (multi electrode array; MEA) and flexible cantilevers to assess cardiac electrical and mechanical function, respectively (Fig. 1a). Only the MEA cardiac chamber was utilized in this study. Following cell attachment with human primary hepatocytes in the liver chamber and patterned human induced pluripotent stem cell derived cardiomyocytes (hiPS-CMs) in the heart chamber, each model was placed on an interval rocker that induced flow by reciprocal levelling between reservoirs resulting in a closed loop recirculating flow ${ }^{12}$. This recirculating serum-free system supports the maintenance of liver (cytochrome (CYP) P450 activity and albumin production) and cardiac function for up to 28 -days ${ }^{15}$. The presence and cellular structure of key hepatocyte (albumin) and cardiomyocyte (myosin heavy chain) markers were confirmed following 14 days by immunofluorescence (Fig. 1b-e). Cardiomyocyte resting membrane potential was shown to be physiologically relevant at approximately -85 to $-90 \mathrm{mV}$, representative of the adult human heart ${ }^{16-18}$. Taken together this provides evidence that the heart:liver model is a suitable system to investigate both parent compound and metabolite driven cardiovascular changes. Importantly the integrated technology also allows in vitro temporal PKPD relationships to be derived.

Value of temporal PK and FPD measurements of parent compound driven changes. To build insight into whether an MPS model could be used to derive temporal PKPD relationships, the well-characterized small molecule, terfenadine was utilized. Terfenadine is a hERG inhibitor resulting in QT prolongation in both preclinical species and in humans, but it is rapidly metabolized, mainly by CYP3A4, to fexofenadine which does not cause QT prolongation ${ }^{19,20}$. The integrated MEA electrodes in both the heart only and heart:liver models were utilized to measure the external field potential. This allows the quantification of FPD, a surrogate of the action potential duration at $90 \%$ repolarization $\left(\mathrm{APD}_{90}\right)$, hence enabling an assessment of the impact of terfenadine on the QT interval ${ }^{21}$.

Heart:liver and heart only models were exposed to $5 \mu \mathrm{M}$ terfenadine or vehicle ( $0.1 \% \mathrm{DMSO})$. Simultaneous heart FPD, beat rate, terfenadine and fexofenadine concentrations in the media and cellular lysates were measured at $0,0.5,1,4,12,16$ and 24 hours. Figure 2 a depicts typical field potential traces obtained from the heart only model. Using these traces FPD and beat rate were quantified, as represented in Fig. $2 \mathrm{~b}-\mathrm{e}$ in the heart only (Fig. 2b,c) and heart:liver model (Fig. 2d,e). In the heart only model, time dependent increases in FPD were noted, with peak measurable changes (8-fold increase) observed at 4 hours. A $5 \mu \mathrm{M}$ nominal concentration of terfenadine in heart only systems had a profound effect on cardiac repolarization at 0.5 to 1 hour that rhythmic cardiac beating ceased and therefore, FPD (Fig. 2b) and beat rate (Fig. 2c) were unquantifiable. These changes in FPD were reduced in the heart:liver model, over the time course of the experiment (Fig. $2 \mathrm{~d})$. This data provides evidence that the FPD responses observed were driven by terfenadine (the parent drug). No changes in cell viability in either the heart or liver chambers at 24 hours were present in either model (Fig. 2f), confirming these changes in FPD were not the result of gross cell death. 
a
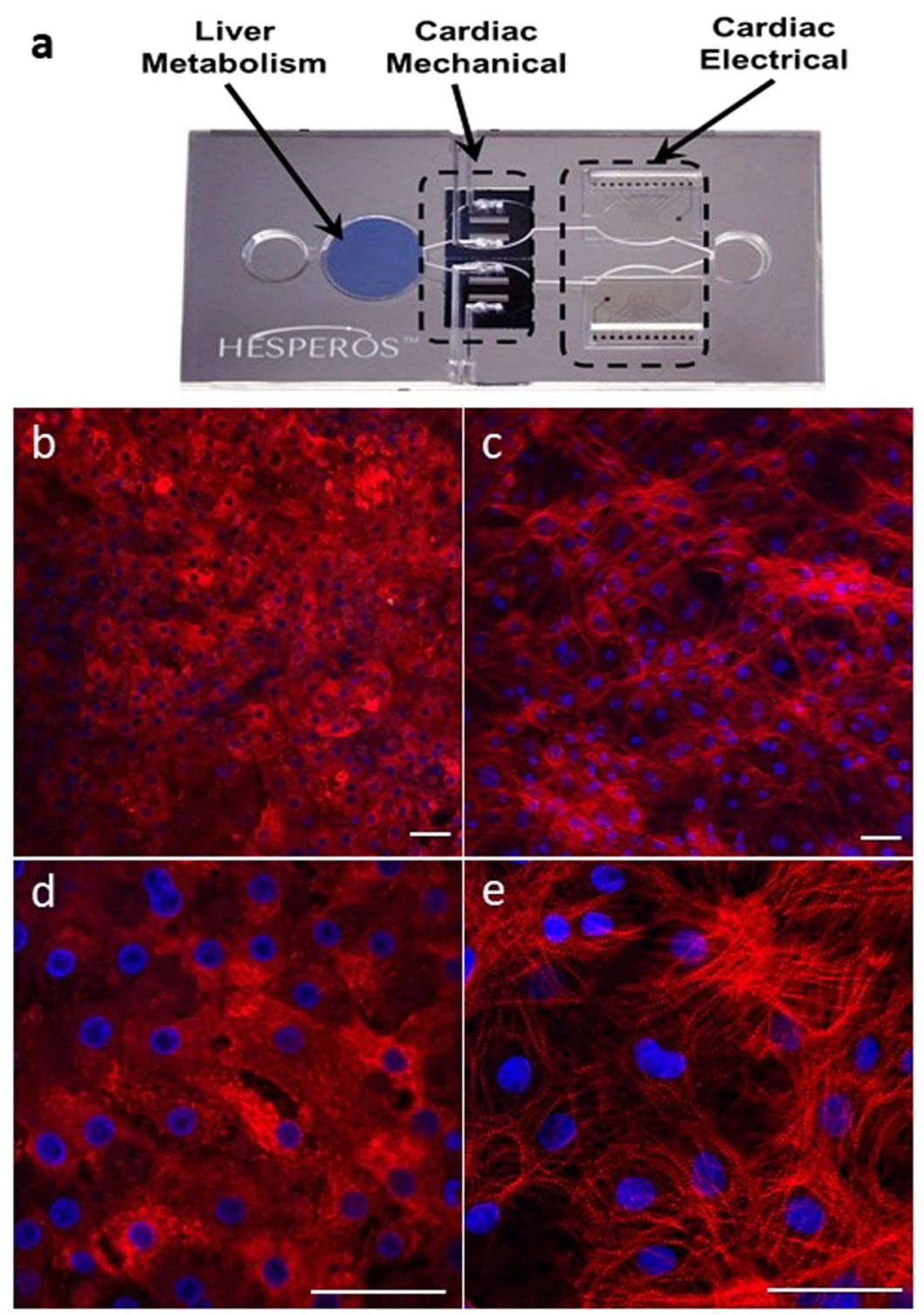

Figure 1. Overview of the heart only and heart:liver MPS models; (a) Photograph of the MPS platform showing the positioning of the 3 cellular chambers ( 1 containing hepatocytes and 2 containing hiPS-CMs) and integrated technology for assessment of cardiac mechanical and electrical activity interconnected via a closed loop recirculating flow system. (b,d) Confocal immunofluorescent images showing primary hepatocytes stained for albumin (red) and nuclei (blue), clearly indicating the cytoplasmic localization. (c,e) Confocal immunofluorescent images showing hiPS-CMs stained for myosin heavy chain (red) and nuclei (blue), indicating the presence of aligned sarcomeres. Scale bars in white are $50 \mu \mathrm{m}$. All images are representative of at least 6 biological and at least 40 technical replicates.

Further evidence that the differences in FPD observed between the heart only and heart:liver models following terfenadine were due to a linked metabolically competent liver, a temporal PKPD relationship was derived by measuring both terfenadine and fexofenadine concentrations in the recirculating media and in cellular lysates of the cardiomyocytes and hepatocytes (Fig. 3). In media from the heart only model, terfenadine concentration also peaked at 0.5 hours, but higher concentrations were measured, $5.88 \mu \mathrm{M} \pm 0.032 \mu \mathrm{M}$ (mean $\pm \mathrm{SD}$ ) and there was limited metabolism to fexofenadine $(0.124 \mu \mathrm{M} \pm 0.003 \mu \mathrm{M} ;$ mean \pm SD) (Fig. 3a). In the heart:liver model, the terfenadine concentration peaked at 0.5 hours at $0.53 \mu \mathrm{M} \pm 0.002 \mu \mathrm{M}$ (mean $\pm \mathrm{SD}$ ) while the fexofenadine concentration increased over the 24 hour measurement period in a time-dependent manner, achieving $3.8 \mu \mathrm{M} \pm 0.075 \mu \mathrm{M}$ (mean \pm SD) at 24 hours (Fig. 3b). This confirms that metabolism of terfenadine occurred when hepatocytes are included. However, loss of terfenadine from media samples was observed by 4 hours in the heart:liver model. This highlights a disconnect between the terfenadine media concentration and FPD response in the heart only model. For example, at the 12-hour timepoint, FPD was increased by 4.8 -fold, but the media terfenadine concentration was only $0.099 \mu \mathrm{M}$. At similar concentrations, but later timepoints in the heart:liver model, no notable increase in FPD was observed. To understand this apparent disconnect between the media terfenadine concentration 

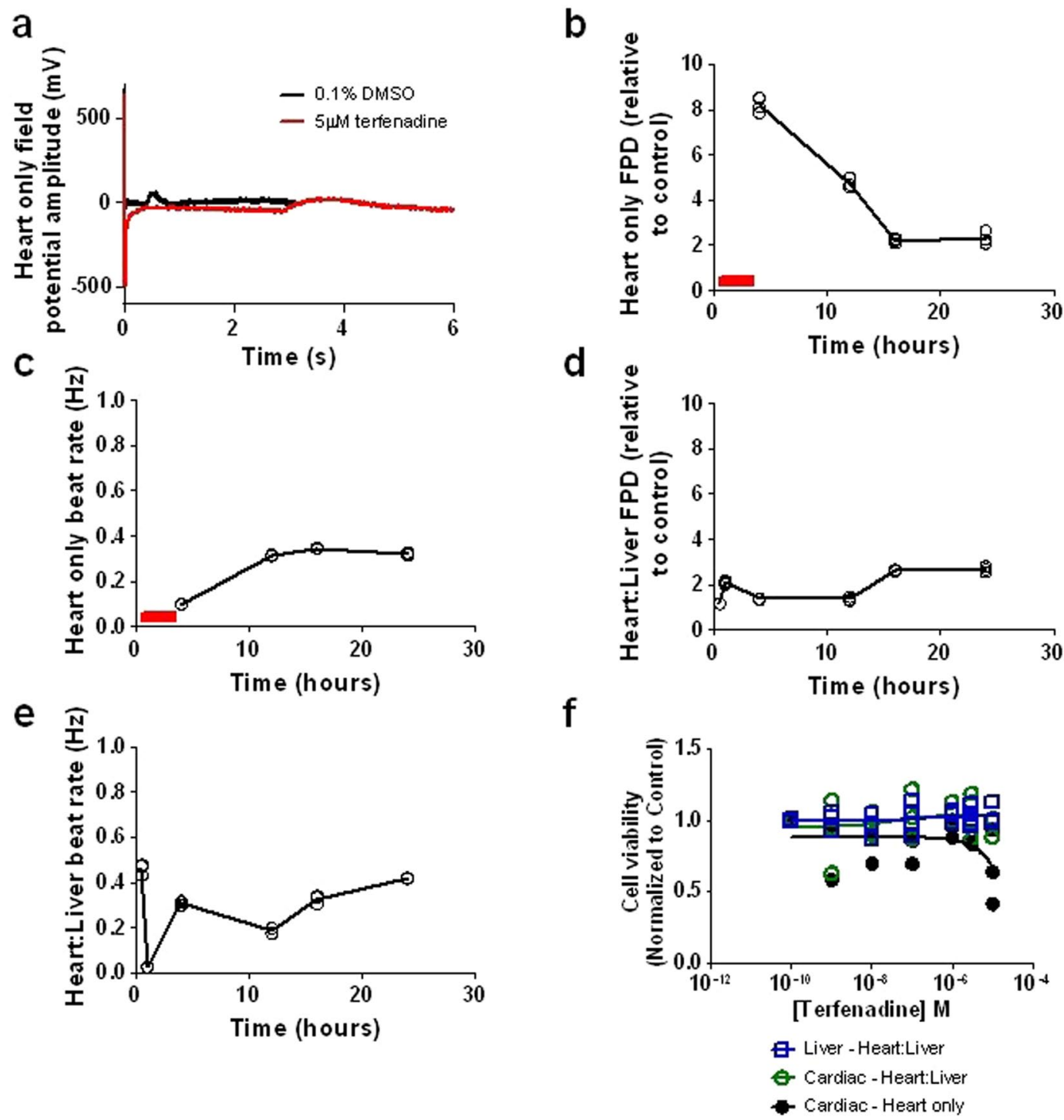

Figure 2. Terfenadine induced PD response in the heart only and heart:liver models; (a) representative field potential traces following $5 \mu \mathrm{M}$ terfenadine (red) or vehicle control $(0.1 \%$ DMSO) (black) at $4 \mathrm{~h}$ in the heart only system. (b) Quantification of FPD and (c) beat rate in the heart only model, 30 second measurements were taken at $0,0.5,1,4,12,16$ and 24 hours, the solid line is the mean of 3 replicates with individual data points plotted. The red bar represents timepoints where no FPD could be quantified due to the arrhythmogenic effects of terfenadine. (d,e) Quantification of FPD and beat rate in the heart:liver model, 30 second measurements were taken at $0,0.5,1,4,12,16$ and 24 hours, the solid line is the mean of 3 replicates with individual data points plotted. (f) Cell viability in both the cardiomyocyte and hepatocyte cellular chambers in both the heart only (black) and heart:liver models (heart: green; liver: blue) following 24 hours of terfenadine, individual data points plotted. All data $\mathrm{n} \geq 3$.

and cellular pharmacodynamic response, cellular lysate concentrations of terfenadine and fexofenadine in each individual cell chamber were assessed (Fig. 3c-e). Within cardiomyocyte lysates from the heart only model, the terfenadine concentration peaked at 4 hours $(2.65 \mu \mathrm{M} \pm 0.016 \mu \mathrm{M}$; mean $\pm \mathrm{SD})$ and decreased over the measurement period which corresponded to the decrease in FPD measured (Fig. 3c). In the cellular chambers of the heart:liver model, terfenadine concentrations peaked between 0.5 and 1 hours, with the accumulation in the liver chamber, $12.04 \mu \mathrm{M} \pm 0.28 \mu \mathrm{M}$ (mean \pm SD), being 10-fold higher to that in the heart chamber, $1.08 \mu \mathrm{M} \pm 0.04 \mu \mathrm{M}$ $($ mean \pm SD) (Fig. 3d-e). Taken together this indicates that the terfenadine-induced change in FPD was driven by the cellular cardiomyocyte concentration (Supplementary Fig. 1). In addition, to the best of our knowledge, this is the first time that an MPS model has been used to derive a quantitative temporal PKPD relationship.

Qualitative and quantitative translational modelling. Qualitatively the terfenadine response was comparable across standard preclinical and clinical cardiovascular safety studies (Table 1). It is important to note that quantitative differences in the magnitude of QT prolongation across pre-clinical species and human 
a

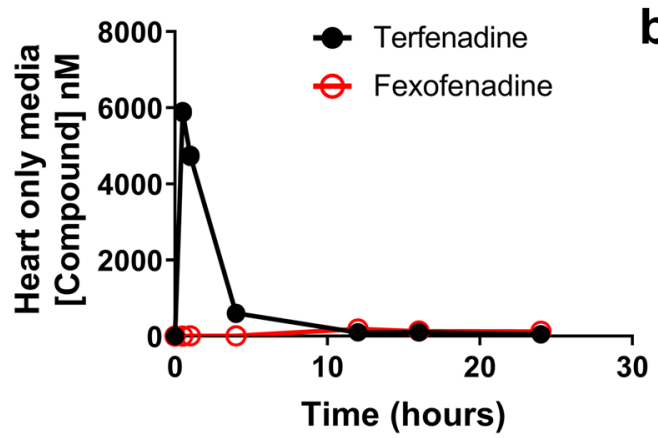

C

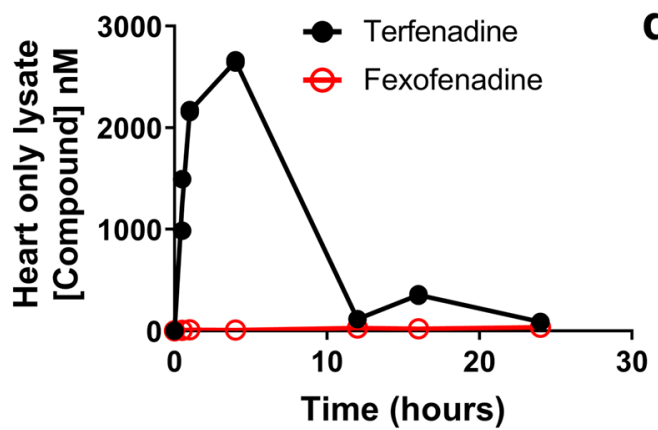

e

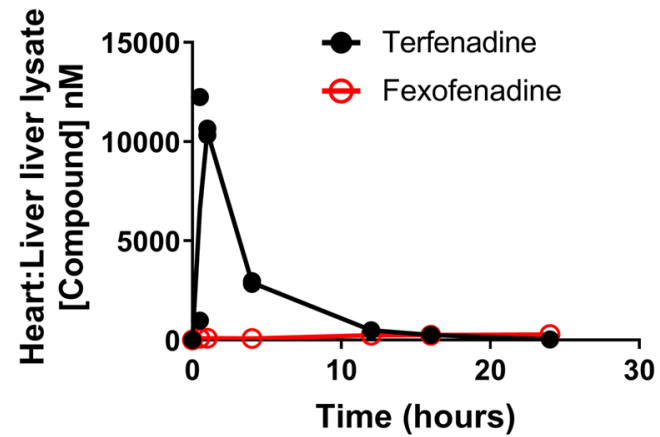

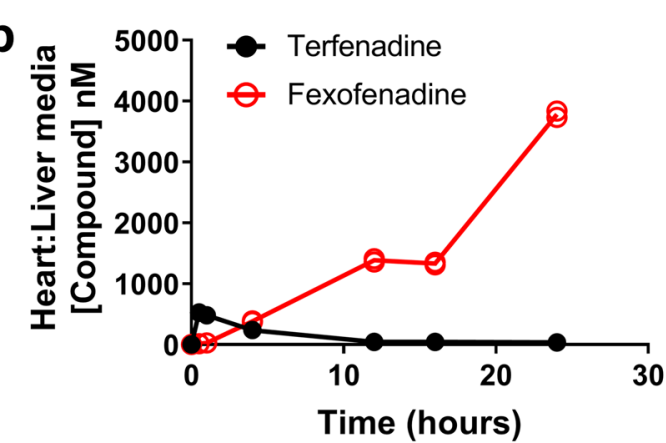

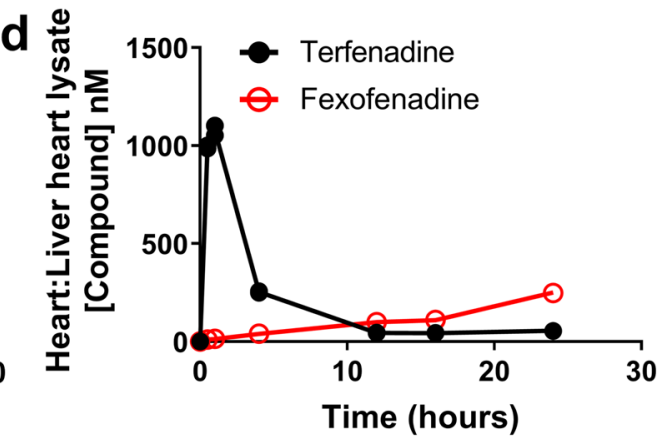

Figure 3. Terfenadine induced PK response in the heart only and heart:liver models. Both terfenadine (black) and fexofenadine (red) concentrations where quantified in the media and cellular lysates following $5 \mu \mathrm{M}$ terfenadine; measurements were taken at $0,0.5,1,4,12,16$ and 24 hours, the solid line is the mean of 2 replicates with individual data points plotted. (a,b) PK profiles in media from the heart only (a) and heart:liver (b) models. (c-e) PK profiles in cellular components from the heart only (c) and heart:liver heart lysates (d); (e) liver lysates MPS models.

is known, the occurrence of QT prolongation can be qualitatively compared, but predicting the magnitude of change from either Guinea pig or dog pre-clinical studies is variable. For example, dogs have been found to be approximately 2.5 times less sensitive to QT prolongation in terms of the magnitude of response ${ }^{22}$.

Due to the measurement of both the PK and PD response simultaneously, a PKPD relationship was developed to predict the FPD changes obtained in these models. To develop a quantitative translational model, temporal data across the MPS platform for terfenadine, fexofenadine, and FPD measurements were used to develop a mathematical PKPD model using Phoenix NLME 7.0 software. The mathematical model could accurately capture terfenadine and fexofenadine exposure levels in the MPS model over time (Fig. 4a and Supplementary Fig. 2). It was further able to capture changes in FPD signals produced by the heart chamber (Fig. 4a). Corresponding peak shifts in cardiac terfenadine PK profiles and FPD profiles verify the assumption that changes in FPD are associated with cardiac bioaccumulation of terfenadine. The goodness of fit captured by the model parameter estimates was further reinforced through percent coefficient of variation (\% CV) rates below 26\% (Supplement Table 1).

FPD simulations were performed based on published in vivo PK profiles to demonstrate the potential of the MPS model to predict cardiotoxicity. Representative PK profiles for Guinea pig $^{23}, \operatorname{dogs}^{24}$ and human ${ }^{25}$ were used as an input for the simulation to predict corresponding cardiac terfenadine bioaccumulation and therefore predicted FPD profiles (Fig. 4b). The predicted FPD values were qualitatively comparable to the reported dog and human QTc prolongation seen in Table 1; however, the FPD predictions failed to reach published Guinea pig QTc prolongation levels (Table 1). The quantitative translation is likely to have been limited by several potential sources of variability; (1) differences between in vitro and in vivo: the chip is made from human cells, and 


\begin{tabular}{|c|c|c|c|c|c|}
\hline Model & Assay/endpoints & Result & Dosing Scheme & Time Frame & Reference \\
\hline Heart only MPS & FPD & $+4.4 \%$ at $1.912 \mu \mathrm{M}$ & $\begin{array}{l}\text { Nominal dose at } \\
\text { time } 0\end{array}$ & $\begin{array}{l}\text { Average observed data } \\
\text { over } 24 \text { hours }\end{array}$ & - \\
\hline Heart:Liver MPS & FPD & $+1.9 \%$ at $0.228 \mu \mathrm{M}$ & $\begin{array}{l}\text { Nominal dose at } \\
\text { time } 0\end{array}$ & $\begin{array}{l}\text { Average observed data } \\
\text { over } 24 \text { hours }\end{array}$ & - \\
\hline $\begin{array}{l}\text { CHO cells } \\
\text { overexpressing } \\
\text { hERG/Kv11.1 }\end{array}$ & $\begin{array}{l}\text { hERG inhibition } \\
\text { (electrophysiology) }\end{array}$ & $\mathrm{IC}_{50} 0.02-0.2 \mu \mathrm{M}$ & $\begin{array}{l}\text { Non-cumulative } \\
\text { concentration } \\
\text { response curve }\end{array}$ & 3-5 minutes & Redfern et al..$^{57}$ \\
\hline $\begin{array}{l}\text { Anaesthetised } \\
\text { Guinea pig }\end{array}$ & QTc & $+8 \%$ at $0.0384 \mu \mathrm{M}$ & $\begin{array}{l}\text { IV infusion for } \\
10 \text { minutes }\end{array}$ & \begin{tabular}{|l|} 
Peak measurements \\
post infusion \\
$(10-40$ minutes $)$ \\
\end{tabular} & Yao et al..$^{23}$ \\
\hline $\begin{array}{l}\text { Anaesthetised } \\
\text { Guinea pig }\end{array}$ & QTc & $+2.8 \%$ at $0.0069 \mu \mathrm{M}$ & $\begin{array}{l}\text { IV infusion for } \\
10 \text { minutes }\end{array}$ & $\begin{array}{l}\text { Peak measurements } \\
\text { post infusion } \\
(10-40 \text { minutes })\end{array}$ & Yao et al..$^{23}$ \\
\hline Dog Telemetry & QTc & $+2.34 \%$ at $0.026 \mu \mathrm{M}$ & $\begin{array}{l}\text { IV infusion over } \\
180 \text { minutes }\end{array}$ & $\begin{array}{l}\text { Average measurements } \\
\text { from } 150-180 \text { minutes }\end{array}$ & Ollerstam et al..$^{24}$ \\
\hline Dog Telemetry & QTc & $+1.72 \%$ at $0.0078 \mu \mathrm{M}$ & $\begin{array}{l}\text { IV infusion over } \\
180 \text { minutes }\end{array}$ & $\begin{array}{l}\text { Average measurements } \\
\text { from } 150-180 \text { minutes }\end{array}$ & Ollerstam et al..$^{24}$ \\
\hline Human & QTc & $+1.5 \%$ at approx $0.004 \mu \mathrm{M}$ & $\begin{array}{l}2 \mathrm{X} \text { daily } 60 \mathrm{mg} \\
\text { dose orally }\end{array}$ & QTc measured day 1-5 & Chen $^{56}$; Pratt et al..$^{55}$ \\
\hline
\end{tabular}

Table 1. Qualitative comparison of the effects of terfenadine in the MPS systems compared to conventional approaches applied during early drug discovery to detect changes in QT prolongation ${ }^{23,24,55-57}$.

although stem-cell models are commonly used to explore FPD, there remains a possibility that the exact response magnitude differs between the chip and in vivo; (2) donor to donor variability has not been accounted for and could potentially be a factor affecting the quantitative responses measured; (3) variability between species: there are clearly different responses observed in vivo between different species and to address the impact of this would require development and validation of species-relevant chip models which is beyond the scope of this manuscript and (4) variability between in vivo responses: the in vivo models do not all agree from study to study, and different observed exposure profiles and FPD could be another confounding layer in the interpretation of the results. Nonetheless, these in vivo simulations show the potential for the MPS model to qualitatively predict cardiotoxicity in humans and in animal models, especially the dog and could be improved to reach quantitative predictions by reducing sources of variability.

Application in drug discovery. Application of MPS model data to build further confidence in the human risk of industrial scientists involved in drug safety disciplines of pharmaceutical companies. Following the characterization of the MPS model with terfenadine and a proprietary AstraZeneca compound (AZ12818677) was evaluated in both the heart only and heart:liver model. Prolongation of the QT interval on the electrocardiogram can lead to a fatal arrhythmia known as Torsade de Pointes ${ }^{26}$. Consequently, it is a mandatory requirement prior to first in human administration of potential new drug candidates that their ability to inhibit the hERG channel, a biomarker for QT prolongation, is investigated. This is typically conducted in vitro at a very early stage in drug discovery because of high-throughput cellular technologies ${ }^{27}$. During the lead optimization phase of drug discovery this in vitro data is supplemented by an in vivo assessment in small animals, typically either the Guinea pig or rat, providing an integrated assessment of cardiovascular safety. These studies allow compounds to be profiled while chemical diversity is available, facilitating the selection of optimal molecules prior to mandated regulatory evaluation of cardiovascular function in non-rodents. AZ12818677 (parent compound) was assessed for hERG inhibition as is standard practice and no quantifiable $\mathrm{IC}_{50}$ value could be derived at concentrations up to $100 \mu \mathrm{M}$. It was concluded that this compound had a low potential to cause in vivo QT prolongation. AZ12818677 was therefore progressed to a small animal cardiovascular in vivo study to assess QT prolongation, in this case the anaesthetized Guinea pig. However, unexpectedly the corrected QT interval increased by $22 \%$ (Fig. 5a) at a concentration of $3 \mu \mathrm{M}$. Due to the lack of activity of AZ12818677 at the hERG channel, it was concluded that the QT prolongation in the Guinea pig was not being driven by AZ12818677-induced hERG inhibition. Subsequent investigations identified a major metabolite, AZ12864610, which was produced in humans and Guinea pig (among other preclinical species). Retrospective analysis of AZ12864610 on the in vitro hERG assay, revealed it was a potent inhibitor with an $\mathrm{IC}_{50}$ of $1.14 \mu \mathrm{M}(95 \%$ confidence interval (CI) $0.86 \mu \mathrm{M}-2.2 \mu \mathrm{M})$. Due to this metabolite driven effect on hERG activity and the in vivo QT prolongation we investigated whether the heart:liver model could prospectively detect this metabolite driven effect (Fig. 5b).

Figure 5c highlights representative FP traces following the parent (AZ12818677) compound in the heart:liver model at 24 hours. These traces were quantified, resulting in an $\mathrm{EC}_{50}$ of $2.7 \mu \mathrm{M}(95 \% \mathrm{CI} 0.9-7.6 \mu \mathrm{M})$ and $13.2 \mu \mathrm{M}$ $(95 \%$ CI 9.8-17.9 $\mu \mathrm{M})$ on FPD in the heart:liver and heart only models, respectively (Fig. $5 \mathrm{~d})$. To confirm the changes in the FPD in the heart:liver model were a result of the formation of the metabolite AZ12864610, it was chemically synthesized and evaluated in the heart only model. As expected, the FPD was prolonged resulting in an $\mathrm{EC}_{50}$ of $1.2 \mu \mathrm{M}(95 \% \mathrm{CI} 0.7-1.9 \mu \mathrm{M})$ (Fig. $\left.5 \mathrm{~g}\right)$. At concentrations above $3 \mu \mathrm{M}$, there were incidents of extreme FPD prolongation (Fig. 5e-f) to the point of arrhythmogenesis suggesting a profound effect on the electrical activity of the cells. This arrhythmogenic activity resulted in large error associated with FPD measurements which were removed from the graphical representation for the sake of clarity (Fig. 5f), but clearly a pharmacodynamic effect was detectable. In addition, cell viability in either the heart or liver chambers at 24 hours was not 

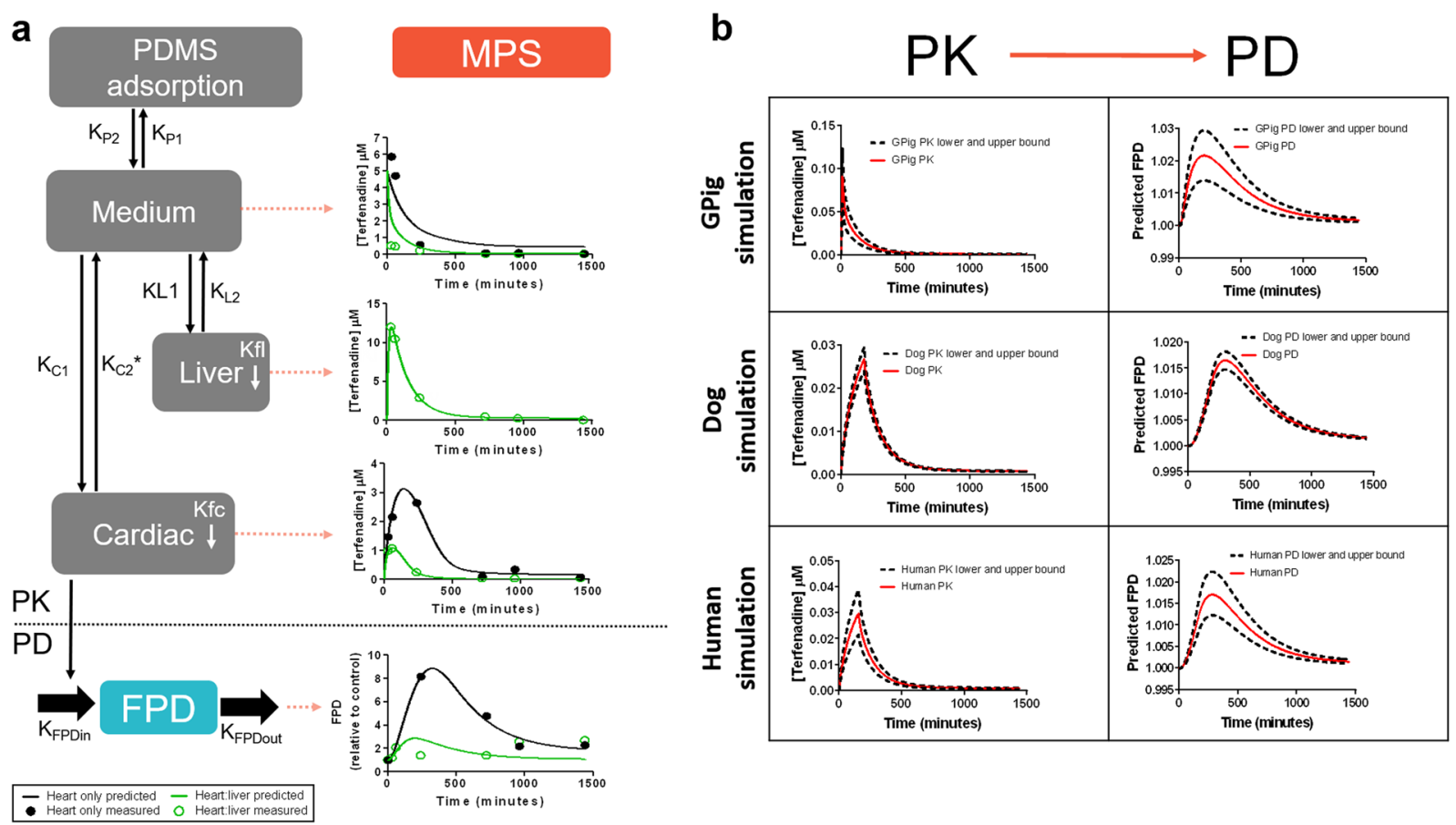

Figure 4. PKPD modelling for the heart only and heart:liver models. (a) Schematic of the modelled system is shown where PDMS, cardiac, and liver compartments allow flow of terfenadine to and from the media compartment. Cardiac and liver compartments are capable of metabolizing terfenadine into fexofenadine and change in FPD is solely dependent on cardiac terfenadine concentration. Terfenadine PK and FPD profiles are plotted for predicted and observed data over 24 hours and are modelled in both heart only and heart:liver systems. Corresponding fexofenadine PK profiles can be found in Supplementary Fig. 2. *Parameter is defined by Eq. 2. (b) Published ${ }^{21,22}$, mean Terfenadine PK profiles \pm SD for Guinea pig, dog and humans were inputted into the model and corresponding mean FPD predicted values \pm SD were simulated.

reduced (Fig. 5h), confirming these changes are not the result of gross cell death. The FPD data obtained for these AstraZeneca compounds and terfenadine demonstrate that these MPS model systems can detect cardiovascular changes driven by either the parent molecule or the metabolite.

The ability of the liver cellular component to metabolize AZ12818677 to AZ12864610 was assessed. The measured concentration of AZ12818677 remained relatively stable over 24 hours. For example, following a nominal concentration of $300 \mu \mathrm{M}, 251 \mu \mathrm{M}(\mathrm{SD} 0 \mu \mathrm{M})$ was measured in the media of the liver component at 0 hours, and $251 \mu \mathrm{M}$ (SD $5.7 \mu \mathrm{M}$ ) was measured at 24 hours (Fig. 6a). Associated with this, only $0.5 \mu \mathrm{M}$ and $1.4 \mu \mathrm{M}$ of AZ12864610 (the metabolite) was detected at 6 and 24 hours respectively (Fig. 6b). Taken together this suggests a complex PKPD relationship, with additional factors influencing the PK and metabolism of the parent compound. Due to the presence of a PD effect in the heart:liver and heart only model despite minimal concentrations of the metabolite, we investigated the potential of cardiomyocytes to produce the metabolite. Since this metabolite is a product of amide hydrolysis (Fig. 6c) it is possible that cardiomyocytes can produce it through cholinesterases ${ }^{28}$. Formation of this metabolite in cardiomyocytes (Fig. 6d) is a significant observation which confirms the PD response in the cardiac only system is derived from a metabolite formed by cardiomyocytes and the response observed in heart:liver model could be a cumulative effect. Although we did not investigate if AZ12864610 undergoes an $\mathrm{N}$-acetylation reaction to form AZ12818677, it could be a possible explanation for the limited detection of the metabolite in incubations with hepatocytes (Fig. 6b), which can potentially convert the metabolite back to parent via $\mathrm{N}$-acetyl transferase enzymes.

\section{Discussion}

Human-on-a-chip models are gaining increasing popularity as tools that will improve preclinical translation thus ensuring potential new drug candidates have a higher probability of success during the clinical trial process. Indeed, the pharmaceutical industry have pointed to defining their context of use to realize their full impact ${ }^{29}$. But to date, these models have been largely characterized only with well-studied reference compounds and so the performance of these models in a 'real-world' setting remains to be determined ${ }^{30,31}$. Moreover, little attention has been paid to exploiting the dynamic nature of these models to derive an understanding of the temporal relationship between measured pharmacodynamic response and pharmacokinetics ${ }^{30}$. The ability to build PKPD relationships in vitro and associated quantitative translational understanding would enable scientists to understand compound behavior prior to in vivo testing, offering a cost and time saving as well as a credible option to reduce and eventually replace animal models. Furthermore, although several groups have developed computational models to analyze data from MPS models ${ }^{7,8,32,33}$, no-one has yet published data on quantitative translation of MPS data to in vivo 
a
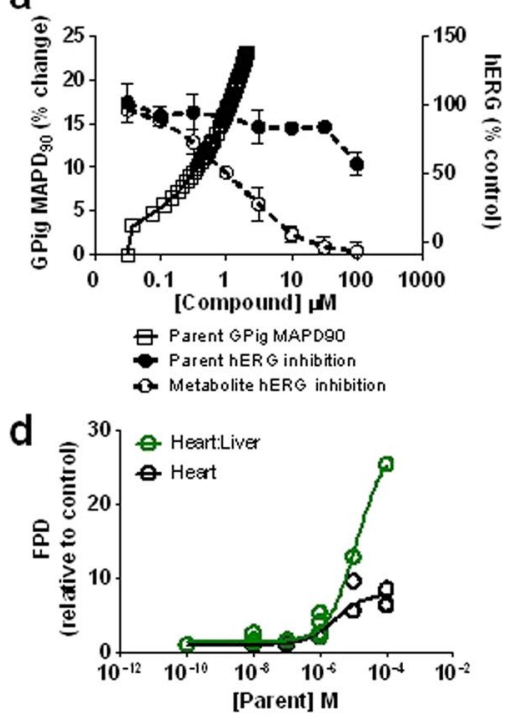

g

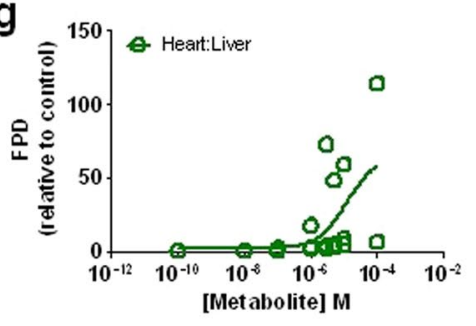

b

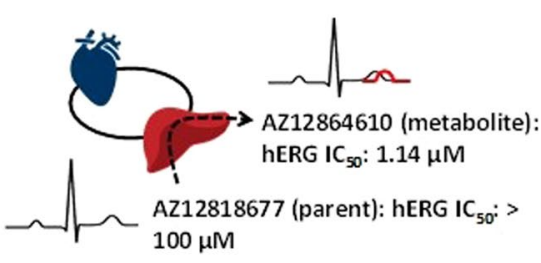

e

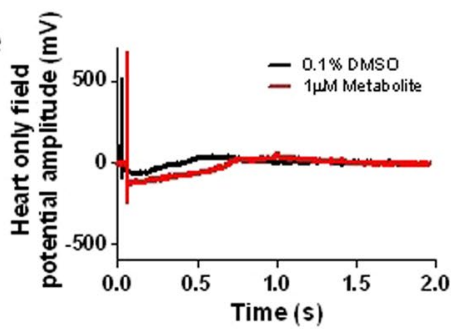

h

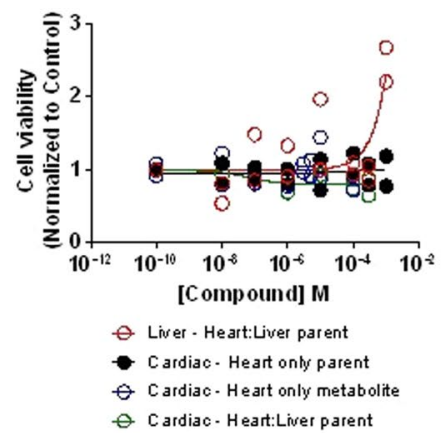

C

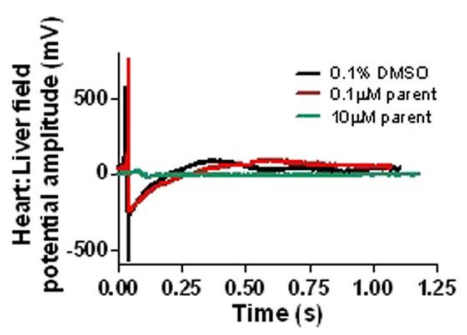

f

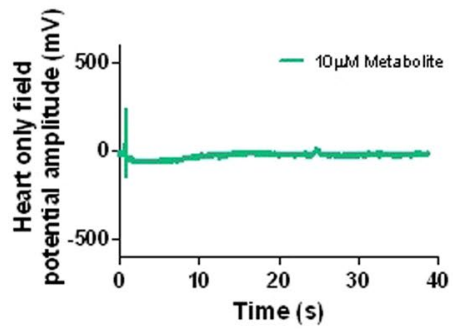

Figure 5. 'Real-world' application of heart:liver models to detect metabolite driven PD effects. (a) Overview of the preclinical in vitro and in vivo QT data for both the parent compound (AZ12818677) and its metabolite (AZ12864610), concentration-effect curves for hERG activity and in vivo QT prolongation in the Guinea pig, $\mathrm{n} \geq 3$. (b) Schematic of AZ12818677 (parent) and AZ12864610 (metabolite) effects on QT prolongation. (c) Representative field potential traces following $0.1 \%$ DMSO (black), $10 \mu \mathrm{M}$ (green) and $0.1 \mu \mathrm{M}$ (red) of the parent compound in the heart:liver model. (d) Concentration-effect curves of the parent compound FPD response in both the heart only (black) and heart:liver (green) models following 24 hours treatment, $\mathrm{n} \geq 3$ (in the heart:liver model at concentrations $\geq 30 \mu \mathrm{M}$, arrhythmic events were observed in two out of three replicates). (e) Representative field potential traces following $0.1 \%$ DMSO (black) and $1 \mu \mathrm{M}$ (red) of the metabolite in the heart only model. (f) Representative field potential traces following $10 \mu \mathrm{M}$ (green) of the metabolite in the heart only model. (g) Concentration-effect curve of the metabolite FPD response in the heart only model, arrhythmias were observed at concentrations $\geq 3 \mu \mathrm{M}, \mathrm{n} \geq 3$. (h) Cell viability in both the heart and liver cellular chambers in both the heart only (parent: black; metabolite: blue) and heart:liver models (heart: green; liver: red) following 24 hours, $n=2$. Individual data points plotted.

outcome whether that be in animals or humans ${ }^{34}$. Here we report first on the use of a heart-liver MPS model to develop a temporal PKPD relationship for the anti-histamine, terfenadine. Using the quantitative data, we then show that we can build a mathematical model that correctly predicts the PK and PD in the MPS, accounting for parent and metabolite concentration across compartments of the device (e.g. conservation of mass) as well as the relationship between target tissue exposure and pharmacodynamic outcomes. Because the model allows the PK and $\mathrm{PD}$ responses to be evaluated separately, we were then able to compare the in vitro $\mathrm{PD}$ response with in vivo outcome in commonly used preclinical species. Finally, we demonstrated the application of this MPS model to drug discovery by recreating the parent and metabolite effects of a proprietary AstraZeneca small molecule compound, which in contrast to terfenadine the cardiovascular effects were driven by the metabolite.

Our simultaneous measurement of cardiomyocyte response to the cardiotoxic drug terfenadine, together with matched quantification of drug concentration in the circulating media and in the lysates of both cardiomyocytes and hepatocytes, show that the pharmacodynamic response was not driven by the concentration of drug in the media but rather by the concentration of drug within the cardiomyocytes. This was also supported by the lack of response of cardiomyocytes to terfenadine in the presence of metabolically competent hepatocytes and the time-dependent increase in the concentration of the hERG-inactive metabolite fexofenadine in the circulating media. Although it is known that terfenadine binds to an intracellular site of $h E R G^{13,14}$, we have for the first time 
a

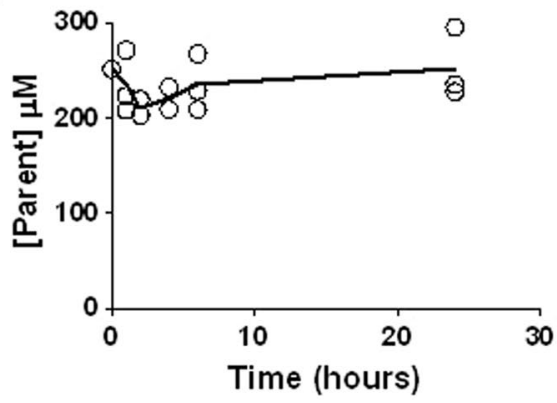

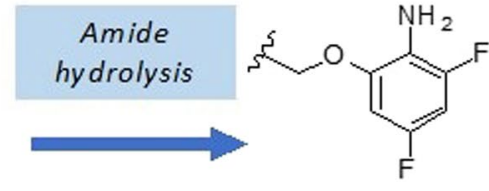

AZ12864610 AZ12818677 Parent Metabolite

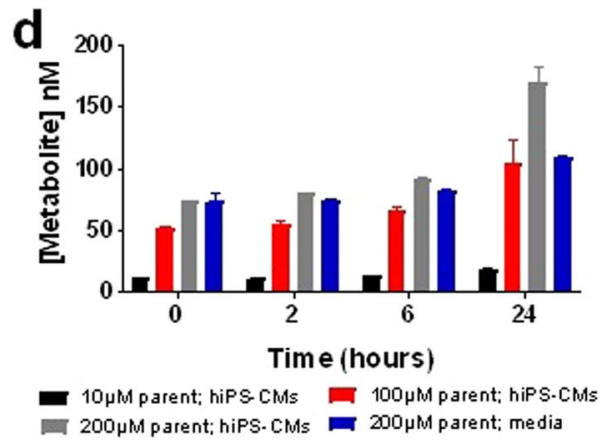

Figure 6. Metabolism of AZ12818677 to AZ12864610. (a,b) Both parent (AZ12818677) (a) and metabolite (AZ12864610) (b) concentrations where quantified in media from the liver compartment following $300 \mu \mathrm{M}$ parent; measurements were taken at $0,1,2,4,6$ and 24 hours, the solid line is the mean of 3 replicates, with individual data points plotted. (c) Metabolic reaction representing de-acetylation/amide hydrolysis of AZ12818677 (parent) to form AZ12864610 (metabolite). (d) Determination of AZ12864610 (metabolite) concentrations at different time points in cardiomyocytes/media following incubations with AZ12818677 (parent).

in a dynamic in vitro system convincingly proven this ${ }^{35}$. With this knowledge, we can determine the peak effect of terfenadine in the model, rather than at a fixed time point ${ }^{36}$. This principle is fundamental to developing an understanding of drug behavior and being able to define this during early drug discovery will be a valuable contribution to the optimization of potential new drug candidates.

The commitment to measure concentration and effect over time enables an accurate description of drug behavior ${ }^{37}$ and is a critical component of quantitative translation. Most of the current in vitro literature assumes that the concentration of drug added to the media drives the effects measured ${ }^{38}$ and that the administered concentration is equivalent to the effective concentration. It has been established that polydimethylsiloxane (PDMS), commonly used in MPS models, absorbs drug compounds, especially those that are highly lipophilic ${ }^{39}$ and measuring actual drug concentration also addresses these limitations. As demonstrated with terfenadine, the PKPD modelling approach was critical for understanding both the flux of compound between compartments as well as the resulting PD response in the context of dynamic exposure profiles of parent and metabolite. Further evaluation of a proprietary AstraZeneca small molecule compound highlights the complexity of changes observed with novel molecules during drug discovery, providing an example of a 'real-world' application. This serves as an illustration of the complexity and diversity of PKPD relationships different compounds can drive. This diversity and ability of both PK and PD effects to occur through both direct and indirect interactions, either instantaneously or delayed further highlights the importance of assessing both the PD and PK effects in conjunction with each other to allow multiple influences on hysteresis to be evaluated ${ }^{40}$.

The application of mathematical modelling of cardiovascular safety data is well established in terms of PKPD modelling of in vivo data to predict and translate human effects at expected therapeutic doses ${ }^{41}$. However, the use of these modelling approaches to MPS data is still in its infancy but has been shown to enhance the value of the MPS models ${ }^{7,42-44}$. Here we demonstrate the potential to use mathematical modelling for translational assessment in the same well-established manner as is commonly applied to in vivo study data. Just as with translating in vivo data across species, the model-derived PK profile and PKPD relationships are built and extrapolated to in vivo scenarios for a single compound at a time by substituting exposure profiles. Further, as with translational validation of in vivo models, proof of concept is initially established with a small number of compounds and confidence in the translational utility of the experimental platform will grow as additional data is generated across differential chemistry, pharmacology and mechanisms. This joint experimental-computation approach provides the prospect of creating quantitative translational understanding for cardiovascular changes based on a human model that can be applied within drug discovery, predicting the preclinical and clinical changes. 
Within this study we have assessed one element of cardiovascular safety: changes in ECG waveforms and intervals. However, drug-induced cardiovascular toxicity can also result in modulation of hemodynamics (heart rate, blood pressure and cardiac contractility) and cardiac pathology ${ }^{45}$. The integrated measurement technologies within this model system (other MEA parameters and flexible cantilevers in combination with a liver module) ${ }^{6,12}$, combined with the ability to collect media samples over time provides the prospect of assessing both changes in cardiac contractility via assessment of force utilizing the flexible cantilevers and pathology via assessment of released soluble cardiac biomarkers, for example, cardiac troponin ${ }^{46}$. Such an approach if validated with a diverse panel of pharmacological agents, and coupled with liver cells of varying metabolic competencies, representing interindividual variability, could enable a holistic assessment of cardiac safety or efficacy in one combined human-based model not only for acute responses, as measured here, but potentially for repeated dose toxicity as cell function can be maintained within these systems for up to 28 -days ${ }^{15}$. This would not only allow detection of changes but potentially provide phenotypic fingerprints of individual compounds to be generated.

In conclusion, our findings serve as a guiding principle to future MPS investigations. Whilst these data are robust, we strongly advocate that further compounds, ideally working across different time frames, through other pharmacological mechanisms and representing a broader chemical diversity, should be evaluated to increase the validation of this heart-liver model but also to build mathematical models that enable quantitative translation to animal and human outcome.

\section{Methods}

Microfluidic housing design and assembly. Microfluidic housings were designed and fabricated as previously described ${ }^{6,7}$ using poly (methyl methacrylate) and PDMS elastomer sheets. The medium recirculates between 2 reservoirs through a microfluidic network formed by the PDMS gaskets, with flow driven by a rocking platform producing a sinusoidal rocking profile with amplitude of $\pm 1^{\circ}$ and period of 60 seconds. The medium flows over the cell-containing areas of the liver coverslips and cardiac cantilever and MEA chips within the microfluidic channel. The system contained a total of $2 \mathrm{~mL}$ of medium throughout the flow channel and reservoirs. Compound dosing was applied at the reservoir closest to the liver chamber, such that the compound would first pass over and interact with the liver before being recirculated throughout the system.

Preparation of chips. Cantilever array chips for force measurements were fabricated following protocols previously described ${ }^{47-52}$. Each cantilever chip contained an array of two rows of microscale cantilevers $(4 \mu \mathrm{m}$ thick, $100 \mu \mathrm{m}$ wide, and $750 \mu \mathrm{m}$ long), with 16 cantilevers per row. Custom patterned MEA chips, for electrical measurements, were fabricated using standard microfabrication procedures following a previously described protocol ${ }^{7}$. Each MEA chip included 10 platinum recording electrodes separated by $1 \mathrm{~mm}$. The surface of each chip were coated with poly (ethylene glycol) (PEG)-containing silane, and this PEG layer was patterned by selective ablation using a $193 \mathrm{~nm}$ ArF excimer laser (Lambda Physik, Santa Clara, CA). The ablated PEG surfaces were coated with human plasma fibronectin (Millipore) diluted in $1 \mathrm{X}$ phosphate buffered saline (PBS) (Thermo Scientific) to concentrations of $10 \mathrm{ug} / \mathrm{mL}$ for MEAs and $50 \mathrm{ug} / \mathrm{mL}$ for cantilevers, then incubated at $37^{\circ} \mathrm{C}$ for 30 minutes followed by a PBS rinse. Printed circuit boards and flexible elastomeric connectors (zebra connectors, FUJIPOLY, $1 \mathrm{~mm}$ wide $\times 18.2 \mathrm{~mm}$ long $\times 9 \mathrm{~mm}$ tall) were incorporated into each system to create an interface between the MEA and a commercially available 60 electrode amplifier (MEA1060, Multichannel-systems).

Cell culture. hiPS-CMs (purchased from Cellular Dynamics) were thawed and seeded directly onto the surfaces at 50,000 cells per MEA and 500,000 cells per cantilever ${ }^{6}$. Primary human hepatocytes were purchased from the Cell Resources Core (Massachusetts General Hospital, Boston, MA, USA). Once thawed, 150,000 hepatocytes were cultured in the hepatic chamber which was coated with rat tail collagen type I $(60 \mathrm{ug} / \mathrm{mL})$ as previously described $^{6}$. The same batch of primary human hepatocytes and hiPS-CMs were used throughout the course of these experiments to block for any potential batch to batch variation within these cells.

Drug preparation. Tefenadine (Sigma Aldrich, T9652, St. Louis MO, USA) and the proprietary AstraZeneca compounds AZ12818677 and AZ10565970 were dissolved in DMSO to a concentration 1000x the desired final nominal dosing concentration. Drug was added through the reservoir closest to the liver chamber to allow for first contact of the drug with the liver. $2 \mu \mathrm{L}$ of the drug stock solution (or vehicle) was added to each system to achieve the desired working concentration in $2 \mathrm{~mL}$ of total volume within the platform.

Cardiac activity as measured by MEA. Custom printed circuit boards were produced to interface the MEAs inside the system with a commercially available 60 electrode amplifier (MEA1060, Multichannel-systems) using a Zebra $^{\circledR}$ elastomeric connector (Fujipoly) ${ }^{7}$. A stimulus generator (STG 1002, Multichannel Systems) was used to stimulate the cells ( $800 \mathrm{mV}$ rectangular pulse, $0.5 \mathrm{~Hz}$ to $3.0 \mathrm{~Hz}$ in $0.25 \mathrm{~Hz}$ increments). The multichannel systems software suite was used to control both the amplifier and stimulator and was used to record action potentials.

Baseline recordings of spontaneous and stimulated cell activity were taken immediately prior to drug application and at $0.5,1,4,12,16$ and 24 hours after exposure to terfenadine and at $0.5,1,2,4$ and 24 hours after exposure to AZ12818677. FPD, spontaneous beat frequency and CV were extracted using Clampfit (Axon Instruments). The FPD was calculated by measuring the time from the initiation of the Q-wave until the apex of $t$-wave. Spontaneous beat frequency was obtained from spontaneous recordings of cardiac activity 30 seconds in length.

Immunofluorescence. Hepatocytes and cardiomyocytes were fixed with $4 \%$ paraformaldehyde for $15 \mathrm{~min}$ utes at room temperature, washed three times with PBS, permeabilized with $0.1 \%$ Triton-X for 15 minutes, then blocked with a PBS solution containing 5\% donkey serum and 1\% BSA for one hour at room temperature. Afterwards, hepatocytes were incubated with sheep anti-human serum albumin primary antibody (Abcam) at 
a 1:100 dilution overnight at $4{ }^{\circ} \mathrm{C}$, washed 3 times with PBS, then incubated with Alexa Fluor 568 conjugated donkey anti-sheep secondary antibody (Thermo Fisher) at a 1:300 dilution for 2 hours at room temperature. Cardiomyocytes were treated similarly with mouse anti-myosin heavy chain primary antibody (Developmental Studies Hybridoma Bank) at a 1:10 dilution, Alexa Fluor 568 conjugated donkey anti-mouse secondary (Thermo Fisher) at a 1:300 dilution. Both cell types were subsequently stained with DAPI (Thermo Fisher) at a $300 \mathrm{nM}$ concentration for 5 minutes. Cells were imaged on a TCS SP8 confocal microscope (Leica Microsystems) and processed using LAS X software.

Cell viability. Hepatocyte viability was assessed via a MTT assay. Briefly, cells were incubated in $500 \mu \mathrm{L}$ of growth medium containing $5 \mathrm{mg} / \mathrm{mL}$ MTT powder for 90 minutes at $37^{\circ} \mathrm{C}, 5 \% \mathrm{CO}_{2}$. The resultant crystals were dissolved in $100 \mu \mathrm{L}$ of lysis buffer (10\% SDS with $0.5 \%$ acetic acid in dimethyl sulfoxide (DMSO)) and was placed into a 96 well plate. Absorbance was read at $570 \mathrm{~nm}$ using a BioTek Synergy HT plate reader. Cardiomyocyte viability was assessed using $10 \%$ solution (v:v) of alamar blue (Thermofisher) in media at $37^{\circ} \mathrm{C}, 5 \% \mathrm{CO}_{2}$ for 4 hours. $100 \mu \mathrm{L}$ of this solution was then placed into a 96 well plate and read at fluorescence excitation wavelength $570 \mathrm{~nm}$ and emission at $590 \mathrm{~nm}$ using the BioTeK Synergy HT plate reader.

Drug determination and quantification with HPLC-MS/MS. Drug concentrations were determined using an LCMS system consisting of an Agilent Technologies (Santa Clara, CA) 1100 HPLC interfaced to an Agilent 6490 Triple Quadrupole Mass Spectrometer with an electrospray inlet. All drugs were separated using gradient elution methodology on a C18 phase (Agilent Zorbax Eclipse Plus, $4.6 \mathrm{~mm}, 100 \mathrm{~mm}$ length, $3 \mu \mathrm{m}$ particle diameter) with binary mobile phase. Samples $(50 \mu \mathrm{L})$ were diluted with acetonitrile and centrifuged to precipitate proteins after addition of the internal standard. The MS was set to positive ion mode and multiple reactive monitoring with nitrogen used for collision induced dissociation. The mobile phase used was $20 \mathrm{mM}$ ammonium formate/acetonitrile for terfenadine and fexofenadine separations and $0.1 \%$ formic acid/acetonitrile for AZ12818677 and AZ12864610. Terfenadine, fexofenadine, AZ12818677 and AZ12864610 were analyzed with an internal standard, propranolol. For terfenadine, fexofenadine and propranolol quantitation, the MRM (parent/ daughter) transitions used were $\mathrm{m} / \mathrm{z} 472$ to 436,502 to 466 , and 260 to 116 , respectively. The collision energy parameter was set to 24 (terfenadine and fexofenadine) and 16 for propranolol. The MRM (parent/daughter) transitions used for AZ12818677 and AZ12864610 quantitation were m/z 466.5 to 449.0 and 424.4 to 406.3, and the collision energy was set to 20 and 16, respectively. For each of the compounds quantified, the dwell time was set to $50 \mathrm{~ms}$ and acceleration voltage to $5 \mathrm{~V}$.

LC-MS/MS analysis of AZ12818677 and metabolite in cardiomyocyte incubations. hiPS-CMs (iCell $^{\mathrm{TM}}$ Cardiomyocytes), cell culture thawing, and maintenance media were purchased from Cellular Dynamics International (cat\# CMM-100-120-005) (Madison, WI). hiPS-CMs were cultured on gelatin coated 96 well plates according to the manufacturers protocol http://www.cellulardynamics.com/products/lit/CDI_iCellCMUsersGuide110927.pdf) at a density of 20000 cells/well. Cell culture media was replaced every 48 hours for 10 days. Following 10 days in culture, hiPS-CMs were treated with 0.1\% DMSO, AZ12818677 (10, 100 or $200 \mu \mathrm{M})$, media and hiPS-CM samples were collated at $0,2,6$ and 24 hours. Concentration of all analytes in the incubations was determined by LC-MS/MS. Acquity ultra performance liquid chromatography [UPLC] system, (Waters, UK) coupled to a triple-quadrupole mass spectrometer (Xevo TQ-S; Waters, Milford, MA) was used to carry out the sample analysis. The analytes were separated by reverse-phase liquid chromatography using Waters Atlantis ${ }^{\circledR} \mathrm{T} 3$, $3 \mu \mathrm{m}, 2.1 \times 50 \mathrm{~mm}$ column (Waters, UK). Mobile Phases A and B consisted of water (containing $0.1 \%$ ammonium formate $\mathrm{w} / \mathrm{v}$ ) and $\mathrm{ACN}$ (containing $0.1 \% \mathrm{FA} \mathrm{v} / \mathrm{v}$ ), respectively. The flow rate was held constant at $0.9 \mathrm{~mL} / \mathrm{min}$ throughout the gradient run. The initial mobile phase composition of $99 \% \mathrm{~A}$ and $1 \% \mathrm{~B}$ was held for $1 \mathrm{~min}$. Mobile phase B was then increased linearly to $70 \%$ until $1.5 \mathrm{~min}$, followed by further increase to $40 \% \mathrm{~B}$ until $3.5 \mathrm{~min}$. At 1.51 min the composition of $\mathrm{A}$ and $\mathrm{B}$ was made to $1 \% \mathrm{~A}$ and $99 \% \mathrm{~B}$ and was held until 4 minutes. At 4.1 min $\mathrm{A}$ and $\mathrm{B}$ were reversed to the initial $99 \% \mathrm{~A}$ and $1 \% \mathrm{~B}$ and held for 1 minute. Analytes quantitation was achieved by MS-MS detection in positive electrospray ionization mode. The MS operating conditions were as following: the capillary voltage was $1.14 \mathrm{kV}$ and source offset was $50 \mathrm{~V}$. The desolvation temperature was set to $600^{\circ} \mathrm{C}$. Nitrogen was used as the desolvation gas $(800 \mathrm{~L} / \mathrm{h})$ and cone gas $(150 \mathrm{~L} / \mathrm{h})$. Argon was used as the collision gas at a flow rate of $0.15 \mathrm{~mL} / \mathrm{min}$. Detection of the ions was performed in the MRM mode using the transitions of $\mathrm{m} / \mathrm{z}$ $424.21 \rightarrow 187.98$ for AZ12864610 and $\mathrm{m} / \mathrm{z} 455.1984 \rightarrow 165.2068$ for verapamil, the internal standard, with a Dwell time of $0.03,0.05$ and 0.02 s, respectively. Peak integration and calibrations were performed using TargetLynx software (Version 4.1, Waters, Milford, MA).

hERG inhibition electrophysiology. A functional electrophysiology assay in CHO cells stably transfected with Kv11.1 (hERG) was conducted on the IonWorks ${ }^{\mathrm{TM}}$ platform as described previously ${ }^{53}$. AZ12818677 and AZ12864610 were tested at $0.01,0.03,0.1,0.33,1,3,10,33$ and $100 \mu \mathrm{M}$ in $0.33 \%$ DMSO. Concentration-effect curves were plotted for each compound and expressed relative to vehicle $(0.33 \% \mathrm{DMSO}) \pm \mathrm{SD}$. The molar concentration of test compound producing $50 \%$ inhibition $\left(\mathrm{IC}_{50}\right)$ were calculated using Graphpad Prism ${ }^{\text {TM }}$ (La Jolla, CA) to fit data to a 4-parameter non-linear regression curve. The maximum (DMSO control) values were fixed. $\mathrm{IC}_{50 \mathrm{~s}} /$ $\mathrm{EC}_{50 \mathrm{~s}}$ were generated in quadruplet for each compound and geometric means and 95\% CI were subsequently calculated.

Anesthetized Guinea pig. This study was ethically reviewed by the establishments' Animal Welfare and Ethical Review Body (AWERB), following the principles of the 3Rs. All work was then conducted under an approved project license, governed by the UK home office under The Animals (Scientific Procedures) Act 1986. All animal identification, conditions of housing, acclimatization, environment, diet and water were in accordance with the 
standard AstraZeneca operating procedures. The effects on cardiac electrophysiology and bioanalysis were assessed in the anaesthetized Guinea pig (Dunkin Hartley) via recording of the ventricular monophasic action potential at $90 \%$ repolarization (MAPD90) as described previously ${ }^{54}$. AZ12818677 or vehicle was tested (I.V.) at 0.744 and $2.015 \mathrm{mg} / \mathrm{kg}$ ( 4 animals per group), formulated as a solution in $30 \% \mathrm{v} / \mathrm{v}$ dimethylacetamide in water (vehicle). Recordings were obtained for 20 minutes following the start of each dose level followed by a 30-minute washout.

Heart only and heart-liver chip PKPD modelling and in vivo simulation. Time course terfenadine and fexofenadine concentration profiles were modelled using a 4-compartment model in Phoenix NLME Software version 7.0. Heart, liver, media, and PDMS compartments and their corresponding parameters can be seen outlined in Fig. 4a. Flow of terfenadine for medium-PDMS and medium-heart pathways was modelled using a first order process as defined by parameters KP1/KP2 and KL1/KL2, respectively. Flow of terfenadine from medium and heart compartments was assumed to be saturable as defined by Eqs 1 and 2. Metabolism of terfenadine to fexofenadine within the heart and liver compartments was modelled using a first order process as defined by parameters $\mathrm{Kfc}$ and $\mathrm{Kfl}$ respectively. It was assumed that there was a delay of fexofenadine release into the system as defined by parameter Kint.

A fifth compartment, FPD, was added to the Phoenix model to represent a PD profile linked to the defined PK model. This PD profile was modelled as a first order process in which the change in effect on FPD was a function of terfenadine heart bioaccumulation as defined by parameters $\mathrm{K}_{\mathrm{FPDin}}$ and $\mathrm{K}_{\mathrm{FPDou}}$. An indirect modelling approach was used for FPD because a hysteresis relationship was found between cardiac terfenadine concentration and FPD values (Supplementary Fig. 1). This approach was further confirmed when \%CV values increased when modelled directly.

Using the experimental data collected (Fig. 3), parameters (except for Kint) were simultaneously fit across heart only and heart-liver systems. Parameters were then fixed and the fexofenadine parameter, Kint, was fit using the experimental fexofenadine concentration data collected (Fig. 3). In the heart only case, the liver compartment was eliminated resulting in relevant parameters being equal to 0 . Volumes of each compartment was defined by system parameters described above. The liver volume was initialized based on the system estimates but allowed to vary to account for any differences between assumed and actual cell numbers or sizes. Once parameters were estimated, the model was used to simulate FPD profiles given published in vivo terfenadine PK profiles. Published Guinea pig $^{23}, \operatorname{dog}^{24}$ and human ${ }^{25}$, PK profiles were used as inputs into the established model in order to predict cardiac bioaccumulation over time, and therefore predict change in predicted FPD. While Guinea pig and dog temporal PKPD data is available in the literature, matching human data is not. Instead, the model made use of a hypothesized dosing schedule that would simulate a PK profile matching published Cmax and AUC data in humans orally dosed with terfenadine, as this information is available in the literature ${ }^{25}$. The reported standard deviation values in PK profile were used to capture the associated error in predicted FPD values. Percent free protein in plasma was assumed to be $2 \%$ for dog and $3 \%$ for Guinea pig and human for the FPD simulation ${ }^{23,24}$.

$$
\begin{gathered}
K_{\text {medium to heart }}=K C 1 \\
K_{\text {heart to medium }}=K C 2 *\left(\frac{1}{1+\frac{\text { Cardiac }_{\text {Terf }}}{I C_{50}}}\right)
\end{gathered}
$$

\section{Data Availability}

All data supporting the findings of this study are available within the article and its Supplementary Information Files.

\section{References}

1. Segre, G. Kinetics of interaction between drugs and biological systems. Farmaco Sci 23(10), 907-18 (1968).

2. Sheiner, L. B. et al. Simultaneous modeling of pharmacokinetics and pharmacodynamics: application to d-tubocurarine. Clin Pharmacol Ther 25(3), 358-71 (1979).

3. Wang, Y. I. et al. Self-contained, low-cost Body-on-a-Chip systems for drug development. Exp Biol Med (Maywood) 242(17), 1701-1713 (2017).

4. Bhatia, S. N. \& Ingber, D. E. Microfluidic organs-on-chips. Nat Biotechnol 32(8), 760-72 (2014).

5. Huh, D. et al. Reconstituting organ-level lung functions on a chip. Science 328(5986), 1662-8 (2010).

6. Oleaga, C. et al. Multi-Organ toxicity demonstration in a functional human in vitro system composed of four organs. Sci Rep 6, 20030 (2016)

7. Oleaga, C. et al. Investigation of the effect of hepatic metabolism on off-target cardiotoxicity in a multi-organ human-on-a-chip system. Biomaterials 182, 176-190 (2018).

8. Sung, J. H., Kam, C. \& Shuler, M. L. A microfluidic device for a pharmacokinetic-pharmacodynamic (PK-PD) model on a chip. Lab Chip 10(4), 446-55 (2010).

9. Esch, M. B. et al. Body-on-a-chip simulation with gastrointestinal tract and liver tissues suggests that ingested nanoparticles have the potential to cause liver injury. Lab Chip 14(16), 3081-92 (2014).

10. Ghanem, A. \& Shuler, M. L. Combining cell culture analogue reactor designs and PBPK models to probe mechanisms of naphthalene toxicity. Biotechnol Prog 16(3), 334-45 (2000).

11. Sung, J. H. \& Shuler, M. L. A micro cell culture analog (microCCA) with 3-D hydrogel culture of multiple cell lines to assess metabolism-dependent cytotoxicity of anti-cancer drugs. Lab Chip 9(10), 1385-94 (2009).

12. Stancescu, M. et al. A phenotypic in vitro model for the main determinants of human whole heart function. Biomaterials $\mathbf{6 0}, 20-30$ (2015).

13. Fernandez, D. et al. Physicochemical features of the HERG channel drug binding site. J Biol Chem 279(11), 10120-7 (2004).

14. Saxena, P. et al. New potential binding determinant for hERG channel inhibitors. Sci Rep 6, 24182 (2016). 
15. Carlota Oleaga, A.L. et al. Candace Martin, Mark T. Schnepper, Arindom Goswami, Reine Note, Jessica Langer, Silvia Teissier, José Cotovio, James J. Hickman, Long-Term Electrical and Mechanical Function Monitoring of a Human-on-a-Chip System. Advanced Functional Materials, 29(8) (2019).

16. Horvath, A. et al. Low Resting Membrane Potential and Low Inward Rectifier Potassium Currents Are Not Inherent Features of hiPSC-Derived Cardiomyocytes. Stem Cell Reports 10(3), 822-833 (2018).

17. Drouin, E. et al. Electrophysiologic characteristics of cells spanning the left ventricular wall of human heart: evidence for presence of M cells. J Am Coll Cardiol 26(1), 185-92 (1995).

18. Hibino, H. et al. Inwardly rectifying potassium channels: their structure, function, and physiological roles. Physiol Rev 90(1), 291-366 (2010)

19. Kamiya, K. et al. Molecular determinants of hERG channel block by terfenadine and cisapride. J Pharmacol Sci 108(3), 301-7 (2008).

20. Rangno, R. Terfenadine therapy: can we justify the risks? CMAJ 157(1), 37-8 (1997).

21. Braam, S. R. et al. Prediction of drug-induced cardiotoxicity using human embryonic stem cell-derived cardiomyocytes. Stem Cell Res 4(2), 107-16 (2010).

22. Parkinson, J. et al. Translational pharmacokinetic-pharmacodynamic modeling of QTc effects in dog and human. J Pharmacol Toxicol Methods 68(3), 357-66 (2013).

23. Yao, X. et al. Predicting QT prolongation in humans during early drug development using hERG inhibition and an anaesthetized guinea-pig model. Br J Pharmacol 154(7), 1446-56 (2008).

24. Ollerstam, A. et al. A novel approach to data processing of the QT interval response in the conscious telemetered beagle dog. J Pharmacol Toxicol Methods 55(1), 35-48 (2007).

25. Honig, P. K. et al. Itraconazole affects single-dose terfenadine pharmacokinetics and cardiac repolarization pharmacodynamics. $J$ Clin Pharmacol 33(12), 1201-6 (1993).

26. Raehl, C. L., Patel, A. K. \& LeRoy, M. Drug-induced torsade de pointes. Clin Pharm 4(6), 675-90 (1985).

27. Pollard, C. E. et al. An introduction to QT interval prolongation and non-clinical approaches to assessing and reducing risk. $\mathrm{Br} \mathrm{J}$ Pharmacol 159(1), 12-21 (2010).

28. Fukami, T. \& Yokoi, T. The emerging role of human esterases. Drug Metab Pharmacokinet 27(5), 466-77 (2012).

29. Ewart, L. et al. Navigating Tissue Chips from Development to Dissemination: A Pharmaceutical Industry Perspective. Exp Biol Med (Maywood)

30. Mathur, A. et al. Human iPSC-based cardiac microphysiological system for drug screening applications. Sci Rep 5, 8883 (2015).

31. Huebsch, N. et al. Miniaturized iPS-Cell-Derived Cardiac Muscles for Physiologically Relevant Drug Response Analyses. Sci Rep 6, 24726 (2016)

32. Tsamandouras, N. et al. Quantitative Assessment of Population Variability in Hepatic Drug Metabolism Using a Perfused ThreeDimensional Human Liver Microphysiological System. J Pharmacol Exp Ther 360(1), 95-105 (2017).

33. Yu, J. et al. Quantitative Systems Pharmacology Approaches Applied to Microphysiological Systems (MPS): Data Interpretation and Multi-MPS Integration. CPT Pharmacometrics Syst Pharmacol 4(10), 585-94 (2015)

34. Cirit, M. \& Stokes, C. L. Maximizing the impact of microphysiological systems with in vitro-in vivo translation. Lab Chip 18(13), 1831-1837 (2018).

35. Vernetti, L. et al. Functional Coupling of Human Microphysiology Systems: Intestine, Liver, Kidney Proximal Tubule, Blood-Brain Barrier and Skeletal Muscle. Sci Rep 7, 42296 (2017).

36. Kitaguchi, T. et al. CSAHi study: Evaluation of multi-electrode array in combination with human iPS cell-derived cardiomyocytes to predict drug-induced QT prolongation and arrhythmia-effects of 7 reference compounds at 10 facilities. J Pharmacol Toxicol Methods 78, 93-102 (2016).

37. Derendorf, H. \& Meibohm, B. Modeling of pharmacokinetic/pharmacodynamic (PK/PD) relationships: concepts and perspectives. Pharm Res 16(2), 176-85 (1999).

38. Abi-Gerges, N. et al. Assessment of extracellular field potential and $\mathrm{Ca}(2+)$ transient signals for early QT/pro-arrhythmia detection using human induced pluripotent stem cell-derived cardiomyocytes. J Pharmacol Toxicol Methods 83, 1-15 (2017).

39. Shirure, V. S. \& George, S. C. Design considerations to minimize the impact of drug absorption in polymer-based organ-on-a-chip platforms. Lab Chip 17(4), 681-690 (2017).

40. Cheung, S. Y. A. et al. A tutorial on model informed approaches to cardiovascular safety with focus on cardiac repolarisation. $J$ Pharmacokinet Pharmacodyn (2018).

41. Collins, T. A. et al. Modeling and Simulation Approaches for Cardiovascular Function and Their Role in Safety Assessment. CPT Pharmacometrics Syst Pharmacol 4(3), e00018 (2015).

42. Prantil-Baun, R. et al. Physiologically Based Pharmacokinetic and Pharmacodynamic Analysis Enabled by Microfluidically Linked Organs-on-Chips. Annu Rev Pharmacol Toxicol 58, 37-64 (2018).

43. Edington, C. D. et al. Interconnected Microphysiological Systems for Quantitative Biology and Pharmacology Studies. Sci Rep 8(1), 4530 (2018).

44. Maass, C. et al. Establishing quasi-steady state operations of microphysiological systems (MPS) using tissue-specific metabolic dependencies. Sci Rep 8(1), 8015 (2018).

45. Laverty, H. et al. How can we improve our understanding of cardiovascular safety liabilities to develop safer medicines? $\mathrm{Br} J$ Pharmacol 163(4), 675-93 (2011).

46. Kopljar, I. et al. Chronic drug-induced effects on contractile motion properties and cardiac biomarkers in human induced pluripotent stem cell-derived cardiomyocytes. Br J Pharmacol 174(21), 3766-3779 (2017).

47. McAleer, C. W. et al. Mechanistic investigation of adult myotube response to exercise and drug treatment in vitro using a multiplexed functional assay system. J Appl Physiol 117, 1398-1405 (2014).

48. Pirozzi, K. et al. Correlation of embryonic skeletal muscle myotube physical characteristics with contractile force generation on an atomic force microscope-based biomicroelectromechanical systems device. Appl Phys Lett 103, 083108 (2013).

49. Smith, A. S. et al. A multiplexed chip-based assay system for investigating the functional development of human skeletal myotubes in vitro. Journal of Biotechnology 185, 15-18 (2014).

50. Smith, A. S. T. et al. Utilization of Microscale Silicon Cantilevers to Assess Cellular Contractile Function in Vitro. J. Vis. Exp. 92, e51866 (2014).

51. Stancescu, M. et al. In Vitro Model of Whole Heart Function. Biomaterials 60, 20-30 (2015).

52. Wilson, K. et al. Measurement of contractile stress generated by cultured rat muscle on silicon cantilevers for toxin detection and muscle performance enhancement. PLoS One 5(6), e11042 (2010).

53. Bridgland-Taylor, M. H. et al. Optimisation and validation of a medium-throughput electrophysiology-based hERG assay using IonWorks HT. J Pharmacol Toxicol Methods 54(2), 189-99 (2006).

54. Morissette, P. et al. The anesthetized guinea pig: an effective early cardiovascular derisking and lead optimization model. J Pharmacol Toxicol Methods 68(1), 137-49 (2013).

55. Pratt, C. M. et al. Dose-response relation between terfenadine (Seldane) and the QTc interval on the scalar electrocardiogram: distinguishing a drug effect from spontaneous variability. Am Heart J 131(3), 472-80 (1996).

56. Chen, C. Some pharmacokinetic aspects of the lipophilic terfenadine and zwitterionic fexofenadine in humans. Drugs R D 8(5), 301-14 (2007).

57. Redfern, W. S. et al. Safety pharmacology-a progressive approach. Fundam Clin Pharmacol 16(3), 161-73 (2002) 


\section{Acknowledgements}

The authors would like to thank Karin Skansen, Matthew Bridgland-Taylor and Karen Philips for conduct of modeling, hERG inhibition experiments and Guinea pig studies on the AstraZeneca compound, respectively. The work at Hesperos was supported by AstraZeneca as well as the National Institutes of Health, SBIR grant number R44TR001326-02, from the National Center for Advancing Translational Sciences (NCATS).

\section{Author Contributions}

A.P., D.P.W., J.J.H. and L.E. conceived the study and were involved in the design of experiments. C.W.M., C.J.L., R.B., B.W., L.R.B., N.N.S., M.T.S., M.S., J.R. and A.S. conducted MPS model experiments or LCMS and analysed the data. R.M., V.P.M., C.J.L. and J.T.M. were involved in the development of the mathematical model and conducted the associated modelling. A.P., L.E., C.W.M., J.J.H., M.L.S. wrote the manuscript which was critically reviewed by D.P.W. and K.F.

\section{Additional Information}

Supplementary information accompanies this paper at https://doi.org/10.1038/s41598-019-45656-4.

Competing Interests: The authors declare no competing interests.

Publisher's note: Springer Nature remains neutral with regard to jurisdictional claims in published maps and institutional affiliations.

(c) (i) Open Access This article is licensed under a Creative Commons Attribution 4.0 International License, which permits use, sharing, adaptation, distribution and reproduction in any medium or format, as long as you give appropriate credit to the original author(s) and the source, provide a link to the Creative Commons license, and indicate if changes were made. The images or other third party material in this article are included in the article's Creative Commons license, unless indicated otherwise in a credit line to the material. If material is not included in the article's Creative Commons license and your intended use is not permitted by statutory regulation or exceeds the permitted use, you will need to obtain permission directly from the copyright holder. To view a copy of this license, visit http://creativecommons.org/licenses/by/4.0/.

(C) The Author(s) 2019 